\title{
Study on Algorithm of Digital Image Watermarking Based on DWT
}

\author{
${ }^{\mathrm{a}}$ Gou xin-ke, ${ }^{\mathrm{b}} \mathrm{Lu}$ ran-ni
}

\author{
${ }^{a, b}$ College of Electrical and Information Engineering Lanzhou Universityof Technology Lanzhou, China \\ Key Laboratory of Gansu Advanced Control for Industrial Processes Lanzhou, china
}

\begin{abstract}
In this article, watermarking algorithm is studied using digital watermarking technology based on the discrete wavelet (DWT) multi-resolution decomposition algorithm. Three-level wavelet decomposition is employed so that images are divided into three serial sub-graphs with high frequency band and one sub-graph with low frequency band. Since low-frequency information is more sensitive to human eyes than high-frequency information, watermarking is embedded into the high-frequency coefficients of the wavelet image. In our experiment, watermarking information is embedded into the original images by Matlab simulation. Besides, salt and pepper noise, Gaussian low-pass filter, amplifying 200\% after narrowing 50\% attack as well as JPEG coding are used to test the algorithm. Results show that our algorithm is robust and the visual effect is satisfactory.
\end{abstract}

Index Terms: Image; MATLAB； Discrete wavelet transform; Robustness

(C) 2011 Published by MECS Publisher. Selection and/or peer review under responsibility of the International Conference on E-Business System and Education Technology

\section{Introduction}

With the development of modern digital technology, illegal operation and illegal authorization of the digital products are increasingly rampant. Illegal use, copyright impersonation and intentional tampering of digital products widely exist in commercial and non-commercial areas, which result in the piracy problem that greatly affects social development. However, traditional protection technology cannot effectively settle these current problems. Aimed at these situations, this paper provides some algorithms, especially the digital image watermarking algorithm based on wavelet transformation, using the imperception[1],robustness[2],security and watermarking. This algorithm attempts to improve the security and robustness of the watermarking information by selecting and judging the embedded position and balances the contradiction between imperceptions and robustness by selecting the embedded intensity factor. This paper embeds the watermarking information into the original images by Matlab simulation and employs many kinds of attack experiments to test the algorithm. Experiments show that watermarking can be well extracted even if many attacks are added, which proves the transparency,robustness, security and easy-to-extract characteristics of the watermarking 
information and realize the unification between theoretical sense and practical sense for watermarking technology.

\section{Digital Watermarking technology}

\subsection{Graphics Wavelet Transform}

\section{1) Wavelet Transform Definition}

According to Wavelet transform, good localization of time and frequency domain, it can characterize a data sets,basic feature to contrast with Fourier transform,so it is the localization of time and frequency domain that can extract information from signal effectively. By stretch and movement computing function to multi-scale detail analysis function and signal. Wavelet transform is called "mathematical microscope", it is the major breakthrough in scientific methods after Fourier transform. In Wavelet function it can get a, b from discrete points. Generally, the scale factor a and translation factor $\mathrm{b}$ discrete sampling as follow. If

$a=a_{0}{ }^{m}, a_{0}>0, \quad m \in Z$

$b=n b_{0} a_{0}^{m} \quad b \square R, n \square Z$

The Wavelet ${ }^{\square_{a, b} t_{\curvearrowright}}$ change to

$\square_{m, n} t \wedge \square a_{0}^{-m / 2} a_{0}^{-m} t \square n b_{0}$

The definition of discrete wavelet transform is

$$
D W T \square \square_{R} f t \square_{m, n} \downarrow t d t
$$

\section{2) Graphics Wavelet decomposition}

DWT Transform Algorithm deposit graphic from independent band and different space,One-dimensional wavelet transform is applied in two-dimensional signals such as image rows and columns may constitute a three wavelet transform[3], after 3 times two-dimensional wavelet transform, the band of graphic like figure 1 show.

\begin{tabular}{|c|c|c|c|}
\hline LL3 & HL3 & \multirow{2}{*}{ HL2 } & \\
\cline { 1 - 1 } LH3 & HH1 & \\
\cline { 1 - 1 } LH2 & HL1 \\
HH2 & \\
LH1 & \multirow{2}{*}{ HH3 } \\
& & \\
\hline
\end{tabular}

Fig1. Three Wavelet Transform

The figure 1 shows after Wavelet Transform, graphic' s energy focus on low frequency sub-band LL,the major information of high frequency sub-band is vertical, horizontal and diagonal's edge information, energy is 
low.

Generally,peoples' eye are sensitive to smooth part of the visual image detail and subtle changes, less sensitive to small changes in Part of the image edges or texture.

Image after sub-band decomposition, Images to the good classification,image edges or texture information mainly to the middle and high frequency detail sub-images of larger value on the wavelet .So it can make a good use of human visual characteristics after use the wavelet transform, this feature is adaptive to the image of the visual mask.

\subsection{Watermark Embed Model}

Watermark Embed rule divided into two types:

Addition Criterion:

$\left.\left.x_{w}\right\lrcorner k_{\curvearrowleft} \square x_{o}\right\lrcorner k_{\curvearrowleft} \square a w k_{\curvearrowleft}$

Multiplication Criterion:

$x_{w} k_{\sim} \square x_{0} k_{-}\left(1 \square a w k_{-}\right)$

Embed Model like figure 2 show:

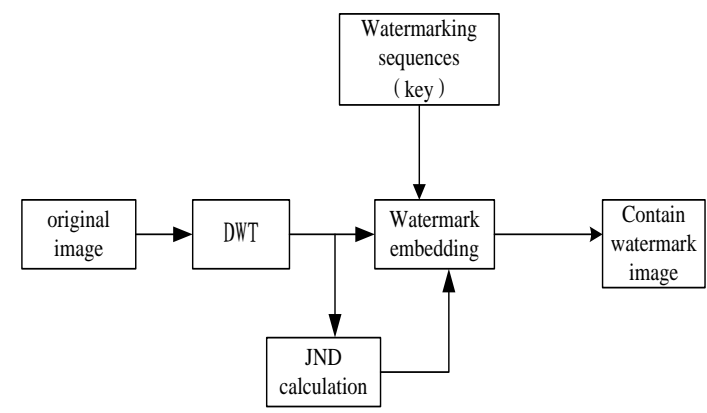

Fig2. HVS-based watermark embed flow chart

Reverse process of extracting the Embed Model like figure 3 show:

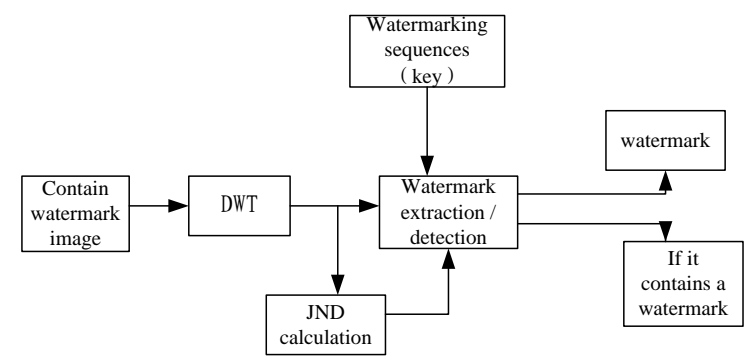

Fig3. HVS-based watermark extract/detect flow chart 


\subsection{The watermark assessment}

\section{1) Peak Signal-to-Noise Ratio}

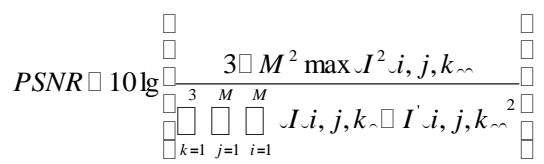

In this formula, "I" means for the $\mathrm{M}^{*} \mathrm{M} * 3$ original image, " $I$ " " means for watermark image.

\section{2) Normalized Correlation}

In order to compare the original watermark signal with extracting watermark signal, by calculate their ratio to compare the similarity between the two signals. If ratio is 1 the two signals are exactly same. For robustness, watermark demand that in signal distortion case can still get the maximum Normalized Correlation. Evaluation formula is:

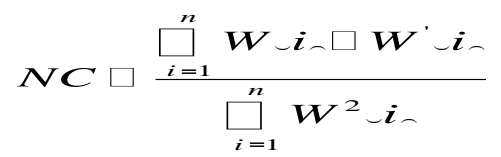

In (6), $W \triangleleft i$-mean the original watermark information which the length is " $\left.n ", W^{\prime}\right\lrcorner i$, mean the extracting watermark information.

\section{Watermark Embedding Algorithm}

\subsection{The steps of watermarking algorithm[5-6] in wavelet domain as follow:}

Step1: Decompose original image use wavelet theory in $\mathrm{L}$ layers, it can get $3 \mathrm{~L}+1$ sub-band,that is $\mathrm{LL}_{\mathrm{L}}, \mathrm{LH}_{\mathrm{I}}$, $\mathrm{HL}_{\mathrm{I}}, \mathrm{HH}_{\mathrm{I}},(\mathrm{i}=1,2, \ldots, \mathrm{L})$. It can get $\mathrm{LH}_{\mathrm{i}+1}, \mathrm{HL}_{\mathrm{i}+1}, \mathrm{HH}_{\mathrm{i}+1}$ by $\mathrm{LL}_{\mathrm{i}}$ decomposition:

Step2: Use improved Arnold transform scrambling the binary watermark image Im, deserve scrambled watermark image $\mathrm{I}_{\mathrm{m}}{ }^{*}$;

Step3: Decompose binary watermark image $\mathrm{I}_{\mathrm{m}}{ }^{*}$ use wavelet theory in $\mathrm{K}$ layers, it can get $3 \mathrm{~K}+1$ sub-band, that is $\mathrm{ll}_{\mathrm{K}}, \mathrm{lh}_{\mathrm{i}}, \mathrm{hl}_{\mathrm{i}}, \mathrm{hh}_{\mathrm{i}},(\mathrm{i}=1,2, \ldots, \mathrm{K})$. If size $\left(\mathrm{LL}_{\mathrm{L}}\right)=\operatorname{size}\left(\mathrm{ll}_{\mathrm{K}}\right)$, then the Ic and $\mathrm{I}_{\mathrm{m}}{ }^{*}$ decomposition to the final graph, $\mathrm{s}$ coefficient matrix is equivalent;

Step4: Generate a random matrix P 0,1 , according to the situation control the numbers of 0,1 . The $\operatorname{size}(\mathrm{P})=$ $\operatorname{size}\left(11_{\mathrm{K}}\right)$;

Step5: If $\mathrm{P}(\mathrm{i}, \mathrm{j})=0, \mathrm{LH}_{\mathrm{L}}(\mathrm{i}, \mathrm{j}), \mathrm{HL}_{\mathrm{L}}(\mathrm{i}, \mathrm{j}), \mathrm{HH}_{\mathrm{L}}(\mathrm{i}, \mathrm{j})$ will not change, then transferred Step7;

Step6: If $\mathrm{P}(\mathrm{i}, \mathrm{j})=1$, then get the value of $\mathrm{LH}_{\mathrm{L}}(\mathrm{i}, \mathrm{j}), \mathrm{HL}_{\mathrm{L}}(\mathrm{i}, \mathrm{j}), \mathrm{HH}_{\mathrm{L}}(\mathrm{i}, \mathrm{j})$ by the following formula

$\mathrm{LH}_{\mathrm{L}}(\mathrm{i}, \mathrm{j})=\mathrm{LH}_{\mathrm{L}}(\mathrm{i}, \mathrm{j})+\alpha^{*} \operatorname{lh}_{\mathrm{K}}(\mathrm{i}, \mathrm{j})$

$\mathrm{HL}_{\mathrm{L}}(\mathrm{i}, \mathrm{j})=\mathrm{HL}_{\mathrm{L}}(\mathrm{i}, \mathrm{j})+\alpha^{*} \mathrm{hl}_{\mathrm{k}}(\mathrm{i}, \mathrm{j})$

$\mathrm{HH}_{\mathrm{L}}(\mathrm{i}, \mathrm{j})=\mathrm{HH}_{\mathrm{L}}(\mathrm{i}, \mathrm{j})+\alpha * \mathrm{\alpha h}_{\mathrm{K}}(\mathrm{i}, \mathrm{j})$

Step7: Repeat Step5, Step6. Get finished until all the points of wavelet coefficient matrix are completed.

Step8: $\mathrm{LL}_{\mathrm{L}}$, other wavelet coefficients of scales did not calculate, random matrix and Watermark strength Factor aconserved as key;

Step9: Use coefficient matrix that is modified to do inverse wavelet transform, reconstruct original image that has the information of watermark.

Simulation results are as follows: 


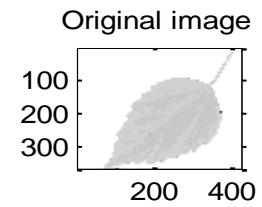

Watermark image

After adding a watermark image

\section{兰州}

Fig4. The simulation graph of embedding algorithm

\subsection{Watermark Extraction and Detection}

Extraction process is the inverse process of embedding process Above-mentioned. According to the Key to use inverse transform, then according to the scope of solutions get Coordinate of Anti-scrambling of Pixels, After do the Anti-scrambling change of extracted Watermark image can deserve the recovery binary watermark image.

\section{Robustness Testing Experiment}

Simulation experiment of this algorithm to watermark, s common anti-attack like Table 1, figure 5 show:

Table 1 The data comparison of simulation results of various attacks

\begin{tabular}{ccccc}
\hline & Untreated & $\begin{array}{c}\text { salt-pepper } \\
\text { nois }\end{array}$ & $\begin{array}{c}\text { Gauss low- } \\
\text { pass filter }\end{array}$ & $\begin{array}{c}\text { amplifying 200\% } \\
\text { after narrowing } \\
50 \% .\end{array}$ \\
\hline PSNR & 35.2047 & 22.2456 & 31.7842 & 22.5261 \\
NC & 1.0000 & $\begin{array}{c}0.7865 \\
\text { ( }\end{array}$ & 0.8979 & 0.8694 \\
$\begin{array}{c}\text { Correspondin } \\
\text { g image }\end{array}$ & (d) & (f) & (i) & (j) \\
\hline
\end{tabular}

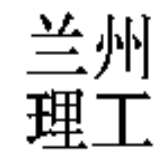

(d)

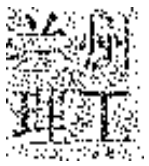

(i)

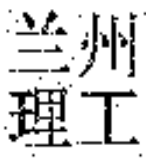

(f)

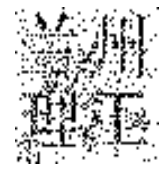

(j)

Fig5. Extracted watermark after attack 
Table 2 compression of JPEG and cropping attack

\begin{tabular}{ccccc}
\hline $\begin{array}{l}\text { Compression } \\
\text { quality factor } \\
\text { for the 40JPEG }\end{array}$ & $\begin{array}{l}\text { Compression } \\
\text { quality factor } \\
\text { for } \\
\text { 80JPEG }\end{array}$ & Cut 1/4 & $\begin{array}{c}\text { Cut } \\
1 / 8\end{array}$ \\
\hline PSNR & 29.9727 & 34.4461 & 18.2137 & 23.1278 \\
NC & 0.8643 & 0.9586 & 0.8523 & 0.9103 \\
$\begin{array}{l}\text { Correspond } \\
\text { ing image }\end{array}$ & $(\mathrm{K})$ & $(\mathrm{l})$ & (m) & (n) \\
\hline
\end{tabular}

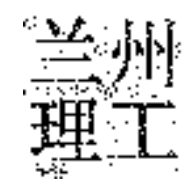

(k)

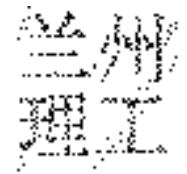

(m)

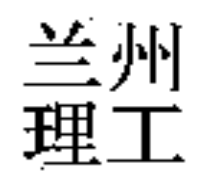

(1)

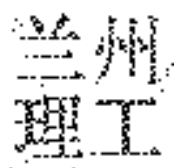

(n)

Fig6. withdraws watermark effect After the JPEG compression and cuts attacks

From experimental results, it can deserve a conclusion that the Digital Watermarking Technology based on Wavelet Transform not only has the merit of good image hiding but also the embedded watermark has strong robustness[7].

\section{Conclusion}

Digital Watermarking Technology across the communication,computer science,cryptography and many other subjects,every area has it's particular research idea. Embed watermark in the image through the method of Wavelet multi-resolution achieve watermark invisibility and robustness conflict well. In the extraction the original image is not required, it well be able to extract the watermark only to know the key. In the embed process, we make the selection and judge of the embed position. Further improve the security and watermark robustness. By MATLAB simulation, do various attack experiment on the image that adding watermark, it also can extract complete watermark. It can solve the problem of copyright protection and piracy, make the implementation watermark technology achieve unity on theoretical and practical.

\section{References}

[1] Zhu Xiaosong, Mao Yaowu, Dai Yaowei, Wang Zhiquan,HVSbased wavelet watermarking scheme[J] Journal of NanjingUniversity of Science and Technology,Jun.2001 Vol.25 No.3:262-268.(in chinese)

[2] Christinel,Wenjunzeng.Image-adaptive watermarking using visual model IEEE journal on selectedareas 
incommunication,1998,16(4):525-539.

[3] Wang Ruihong, Wavelet-based digital watermarking algorithm, North China Electric Power University, a master's degree thesis, November,2007.(in chinese)

[4] Zhang Xiaofeng,Duan Huilong, Wavelet-based Image Watermarking Method[J],Computer Engineering and Application,November 2004.(in chinese). (in chinese)

[5] Zhang Hanling, MATLAB in Image Processing, Tsinghua University Press[M], May 2008 version 1, P280-293. (in chinese)

[6] Wang Xiangyang, Hou Limin, Wu Jun, Intensity based on image feature points robust digital watermark embedding scheme[J] Automation Technology, 2008, No. 34 (1 st Edition): Pl-6. (in chinese)

[7] Cheng Zhengxing,Wavelet analysis and application[M]XI,AN Jiaotong university press, July 2000 Version 3, P58-64. (in chinese) 